\title{
New applications of Spectral Edge image fusion
}

\author{
Alex E. Hayes ${ }^{\mathrm{a}, \mathrm{b}}$, Roberto Montagna ${ }^{\mathrm{b}}$, and Graham D. Finlayson ${ }^{\mathrm{a}, \mathrm{b}}$

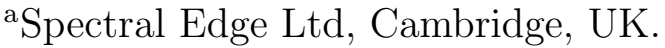 \\ ${ }^{\mathrm{b}}$ University of East Anglia, Norwich, UK.
}

\begin{abstract}
In this paper, we present new applications of the Spectral Edge image fusion method. The Spectral Edge image fusion algorithm creates a result which combines details from any number of multispectral input images with natural color information from a visible spectrum image. Spectral Edge image fusion is a derivative-based technique, which creates an output fused image with gradients which are an ideal combination of those of the multispectral input images and the input visible color image. This produces both maximum detail and natural colors.

We present two new applications of Spectral Edge image fusion. Firstly, we fuse RGB-NIR information from a sensor with a modified Bayer pattern, which captures visible and near-infrared image information on a single CCD. We also present an example of RGB-thermal image fusion, using a thermal camera attached to a smartphone, which captures both visible and low-resolution thermal images. These new results may be useful for computational photography and surveillance applications.
\end{abstract}

Keywords: image fusion, Spectral Edge, near-infrared, thermal, sensor fusion, surveillance, real-time, image processing

\section{INTRODUCTION}

Many image acquisition systems rely upon multiple sensors that respond to different bands of electromagnetic radiation, such as visible light, ultraviolet light, and thermal radiation. The most common example would be a standard digital camera sensor, which is sensitive to visible light in three visible bands, corresponding to red, green and blue. In more complex systems, such as satellite imaging, there can be dozens of sensors that capture images each at a different band, raising the issue of how to efficiently display all the information for a human operator - or in a way that is easy to represent on available display technologies. This is the problem image fusion tries to solve.

Image fusion usually involves combining one or more input images, or image channels, and producing an output composite image with the most salient details transferred from each input image and combined. Typically some way of representing image details is chosen, and then a weighting factor is applied to each input image, either globally or locally. There are a wide variety of applications for image fusion, including remote sensing, ${ }^{1}$ medical imaging, ${ }^{2}$ multifocus image fusion, ${ }^{3}$ RGB-NIR image fusion, ${ }^{4}$ and surveillance. Any situation where multiple imaging modalities are used simultaneously has the potential to utilize image fusion to provide a more compact representation for human perception. Color to greyscale is also a related band reduction problem, where we seek to represent three-dimensional color information in one greyscale dimension. ${ }^{5}$

Widely used image fusion methods include methods based on the discrete wavelet transform (DWT), ${ }^{6}$ pyramidal techniques such as the ratio of low pass pyramid (ROLP), ${ }^{7}$ neural networks, ${ }^{8}$ and derivative-based approaches such as that of Socolinsky and Wolff (a precursor to Spectral Edge fusion). ${ }^{9}$ There are also a wide variety of lesser known image fusion methods, with a great deal of recent research in this area.

Typically the output image of these image fusion methods is a greyscale image - when a color output image is required, a new luminance channel is produced and then combined with the color information of the input image (for example using a luminance-chrominance decomposition). Spectral Edge image fusion works in a different

Further author information: E-mail: alex.hayes@spectraledge.co.uk, r.montagna@spectraledge.co.uk, g.finlayson@uea.ac.uk 
way. Color information is built into the method, creating an output image which simultaneously transfers detail and maintains color integrity. For more information about the mathematical background to Spectral Edge image fusion, see the work of Connah et al. ${ }^{10}$ The Spectral Edge image fusion method has been used for RGB-NIR (visible and near-infrared) image fusion, ${ }^{11}$ remote sensing, and RGB-thermal surveillance image fusion.

In this paper, we detail new applications of the Spectral Edge image fusion method. We first use a sensor with a modified Bayer pattern to capture RGB and NIR images simultaneously (unlike previously, where the RGB and NIR images were captured separately, with a modified DSLR camera), then fuse them into an improved output RGB image. We then show the results of fusing RGB and thermal images captured using the FLIR ONE thermal camera accessory for smartphones, represented as both natural color and false color.

\section{BACKGROUND}

\subsection{The color structure tensor}

Di Zenzo introduced the color structure tensor, ${ }^{12}$ which represents the relation between the gradients in the $x$ and $y$ directions across multiple image channels, and gives a direction of maximal contrast at a particular image pixel. The Jacobian of an N-channel multispectral image is defined as the combined gradient vectors from each channel:

$$
J=\left[\begin{array}{cc}
\frac{\partial I_{1}}{\partial x} & \frac{\partial I_{1}}{\partial y} \\
\frac{\partial I_{2}}{\partial x} & \frac{\partial I_{2}}{\partial y} \\
. . & . . \\
\frac{\partial I_{N}}{\partial x} & \frac{\partial I_{N}}{\partial y}
\end{array}\right]
$$

Where $I_{n}$ is the nth input channel, out of $N$ input channels.

The structure tensor - sometimes called the first fundamental form - is defined as the inner product of the Jacobian:

$$
Z=J^{T} J
$$

The $2 \times 2$ structure matrix $Z$ has the property that it encodes the multispectral gradient magnitude in all image directions.

Socolinsky and Wolff (SW) made the observation that using the eigendecomposition of the structure tensor we can solve for the $\vec{V}$ where there is a maximum change in the underlying image and the magnitude of this change. Their idea then was to compute the direction and magnitude of maximum change at each point of the image, creating a single gradient field (from the $N$ gradient fields in (1)). In fact we cant quite do this directly as the calculated $\vec{V}$ is only unique up to a sign. SW adjusted this sign to be the same as that of the gradient in this direction of the mean image (over the $N$ input channels).

\subsection{Gradient field reintegration}

Let us denote the gradient field derived via Socolinsky and $W_{0 l f f}^{9}$ as $\nabla G$. It could be that there is no image that has derivatives equal to the ones we seek. After all, for every pixel we have an $x$ and $y$ derivative yet the reintegrated image has a single pixel value. Thus, the typical away to solve this reintegration problem is to solve the Poisson equation

$$
\underset{O}{\arg \min }\|\nabla O-\nabla G\|
$$

In finding the image $O$ it is often the case that the reintegrated image has details not in any of the original $\mathrm{N}$-image planes. Indeed $O$ will typically have haloes and/or bending artifacts. The gradient field is not integrable and in solving for $O$ (in a least-squares sense) the error manifests itself in these visible artifacts. 
One way to remove artifacts from the reintegrated image is to place a constraint on $O$. Let us denote all images that are a a linear combination of the $N$-image planes as

$$
O \in P_{1}(I)
$$

Or, if we also allow second order polynomial terms (for an RGB image this would be $R^{2}, G^{2}, B^{2}$ and $R G$, $G B$ and $B G$ ) we write

$$
O \in P_{2}(I)
$$

where $P_{n}$ denotes the order of the polynomial expansion. Finlayson et al. ${ }^{13}$ proposed solving for $I$ as

$$
\underset{O \in P_{2}(I)}{\arg \min }\|\nabla O-\nabla G\|
$$

\subsection{Spectral Edge image fusion}

The Spectral Edge (SE) method is a derivative domain image fusion technique. It is based on the color structure tensor, but instead of solving for a greyscale output image with gradient as close as possible to the ideal SW gradient, it finds an output color image whose structure tensor is equal to the Socolinsky and Wolff structure tensor, meaning it contains the most important details, while also remaining as close as possible to that of the input RGB image, meaning its color remains the same. ${ }^{10}$

Mathematically, we find a new Jacobian $\nabla R$ (per pixel) such that

$$
\underset{\nabla R}{\arg \min } \| \nabla R-\tilde{\nabla R \|} \text { s.t. } K^{T} K=J^{T} J
$$

Where $J$ is the Jacobian of the input high-dimensional image $H, K$ the Jacobian of the output RGB image $R$, the input color gradient is defined as $\tilde{\nabla R}$, and the final output image gradient as $\nabla R$. Effectively this means finding a $2 \times 2$ rotation matrix to transform input RGB gradients into fused RGB gradients, while maintaining maximum detail, and as close a color as possible to the input RGB. Details of how to solve this minimization can be found in Connah et al. ${ }^{10}$ Typically $\nabla R$ is a 3 -dimensional gradient field (for a color image). The individual color planes are again found by look-up-table reintegration. ${ }^{13}$

A further development of this method, iterative Spectral Edge fusion, has the potential to increase its effectiveness at the cost of a slight increase in computational speed. ${ }^{11}$ It repeats the fusion process, using the output of the previous iteration as the guide RGB image for the next iteration, producing a stronger effect - but too many iterations may produce unnatural results.

\section{IMAGE FUSION USING AN RGB-IR BAYER PATTERN}

We have implemented Spectral Edge image fusion using raw sensor data, captured from an Omnivision OV4682 sensor, ${ }^{14}$ as shown in fig. 1. This 4 -megapixel sensor has a modified Bayer pattern, with one of the green pixels in each $2 \times 2$ region replaced with a near-infrared pixel (creating the pattern [R G; NIR B].

This sensor allows the acquisition of perfectly-registered RGB and NIR image data - previous RGB-NIR image fusion research has used images captured using a standard camera with the hot mirror removed, and different filters placed in front of the camera ${ }^{4}$ (the largest data set of this kind is the EPFL RGB-NIR data set ${ }^{15}$ ). This previous method has the problem of objects and the camera moving between the separate acquisitions, resulting in misregistration, leading to artifacts in the fused images. The proposed method avoids these problems.

The Omnivision sensor only provides RAW sensor data or an output RGB image, so to perform image fusion we created our own custom image pipeline. We first created a demosaicing algorithm based on Pixel Grouping ${ }^{16}$ (one of the demosaicing methods available in the open source raw image reader 'dcraw'), but customized for the different RGB-IR Bayer pattern. 


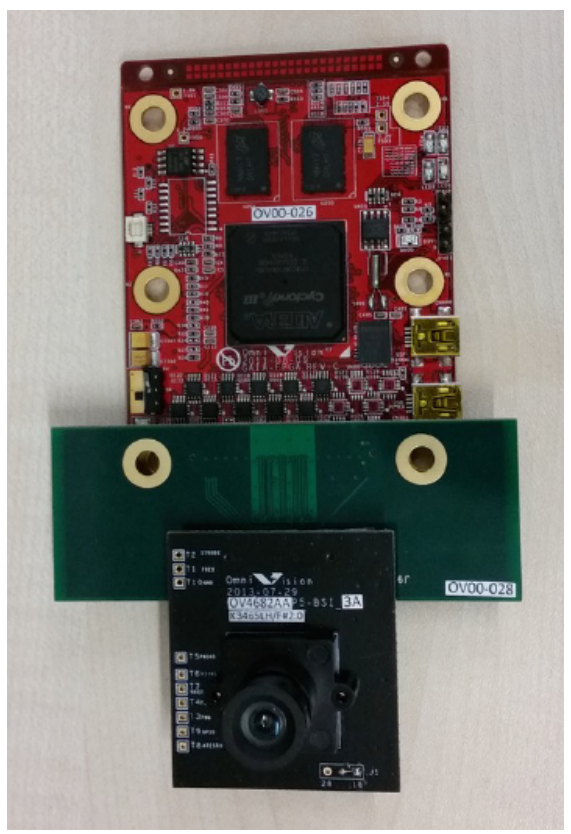

Figure 1: Omnivision OV4682 sensor

We took images of an X-rite ColorChecker Digital SG (140 color patches) with the OV4682 sensor at different exposure levels, and then acquired rendered RGB images of the same scene using a Canon Powershot G11 camera, two examples of which are shown in 2 . We registered these images, and used them to create a custom color correction matrix, optimized for image fusion.

For white balance, we used the Shades of Grey algorithm, ${ }^{17}$ which combines the Max-RGB and Grey-World algorithms to find an optimal mid-point between the two extremes. Finally, we create an attractive RGB image, which uses only the visible spectrum information, and a greyscale NIR image, which only uses the near-infrared sensor data. Once we form full-resolution RGB and NIR images, we then apply the Spectral Edge image fusion algorithm to fuse them, and produce a new RGB image with additional detail and superior image quality.

Figs. 3 and 4 shows example outputs of our image pipeline. The RGB image (a) is constructed using only visible spectrum information, and can be considered an approximation of the image a typical camera would produce of the scene, while the NIR image (b) only uses the near-infrared intensity to construct the image. The central bush in fig. 3 appears dark and lacking in detail in the RGB image, but additional details are visible in the near-infrared - the chlorophyll present in vegetation has a far higher reflectance in the near-infrared than in the visible spectrum. A similar effect is visible in fig. 4. The SE fusion result (c) is superior in both cases to the original RGB image, as the near-infrared details are transferred, while maintaining natural colors. These examples show quite a typical image scenario in which SE fusion can dramatically improve image quality.

\section{RGB-THERMAL IMAGE FUSION USING THE FLIR ONE}

The FLIR ONE is a thermal camera accessory for smartphones, with $160 \times 120$ thermal resolution. ${ }^{18}$ It has both visible RGB and thermal cameras, and is capable of exporting both modalities separately as well as fusing them with its own patented method. ${ }^{19}$

We used the FLIR ONE to capture visible and thermal images, and then applied the Spectral Edge algorithm to produce a color output image. For this application we used the iterative Spectral Edge variant, which produces stronger results. ${ }^{11}$ In the FLIR fusion patent, they assert that standard fusion methods such as SE are not preferred because "results are generally difficult to interpret and can be confusing to a user since temperature data from the IR image, displayed as different colors from a palette or different greyscale levels, are blended with color data of the visual image", but we show here that the results of combining visible colors and thermal detail can be useful and interesting. As an alternative fusion result, one more similar to the MSX technology used by 


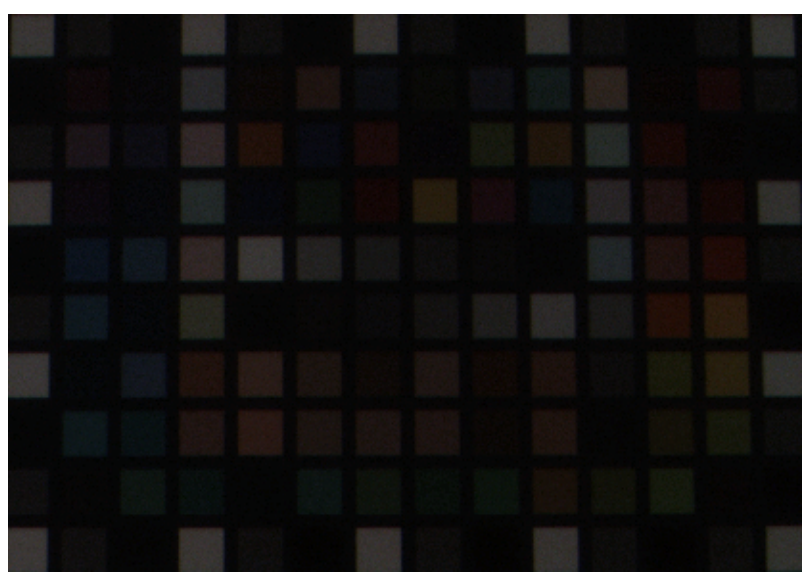

(a) OV4682 - dark

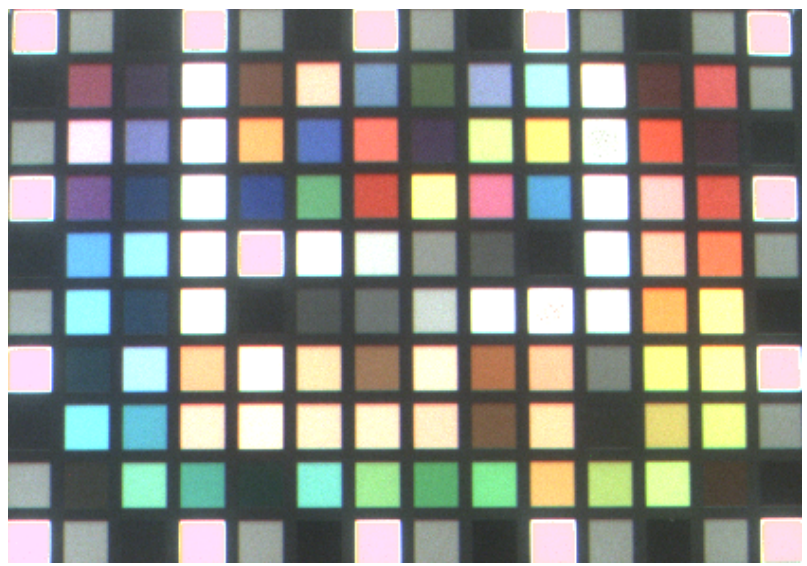

(c) OV4682 - bright

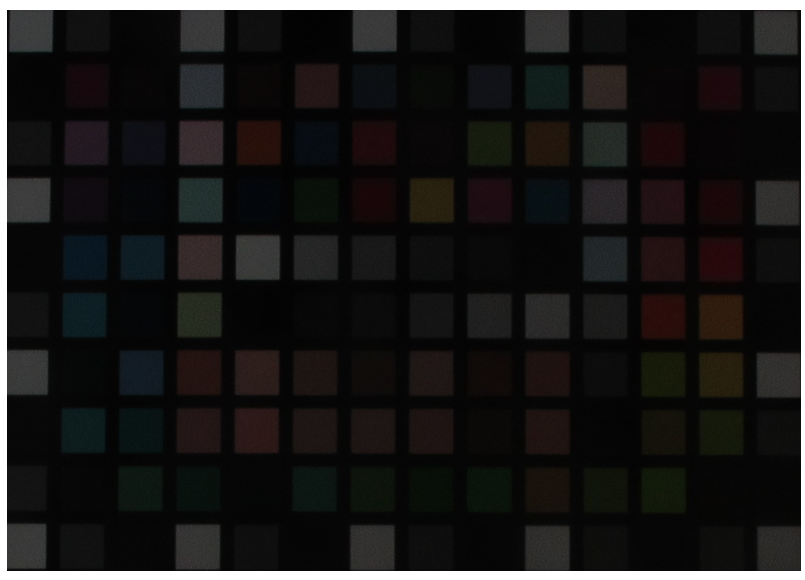

(b) Canon Powershot G11 - dark

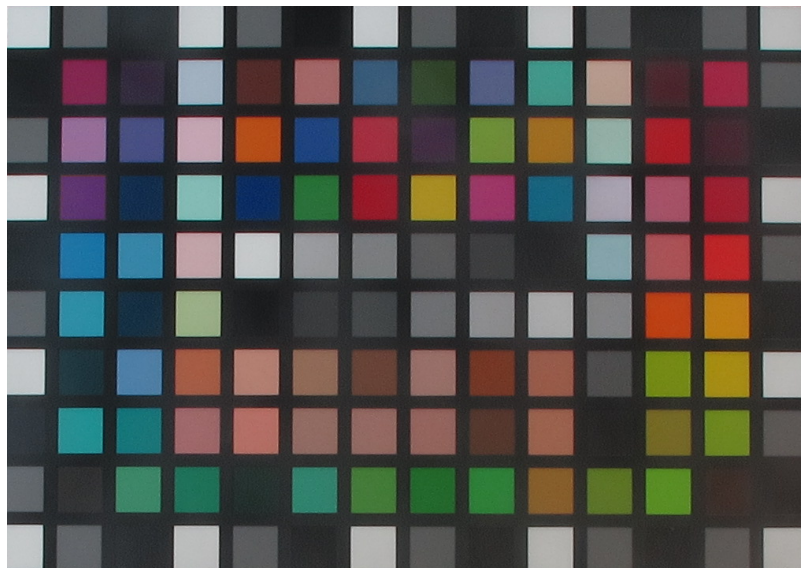

(d) Canon Powershot G11 - bright

Figure 2: $\mathrm{X}$-rite ColorChecker Digital SG - color correction images

FLIR, we take the false color from the thermal image, and use this as the color input for SE fusion, with the luminance channel of the RGB image used as an additional detail input.

In figs. 5, 6, and 7, we show three example scenes. In each scene, we show the RGB image taken by the FLIR ONE visible spectrum camera in (a), the greyscale thermal image in (b), and the SE fusion result, the RGB image enhanced with the thermal image information in (c). We then show the false color thermal image in (d), the fused false color image produced by FLIR MSX technology in (e), and our alternative fused false color image in (f), with the false color thermal image used as our RGB input, and the visible spectrum image used to enhance its detail.

The first result, fig. 5, shows a scene of several parked cars. The nearest car is considerably warmer than the other cars, perhaps having been recently used, and this heat is transferred into the natural color SE fusion result (c) as extra brightness compared to the original. The water cooler in fig. 6 shows high thermal readings in the center of the cooler, due to the heat of the cooling mechanism. This heat is effectively shown in the natural color fusion result (c), as a warm glow. The third scene is a night scene, with a boat full of rowers hidden in the darkness in the visible image, but their body heat is visible in the thermal image. The natural color fusion result shown in fig. 7c shows somewhat unnatural colors, due to the extremely dark visible RGB image, lacking color information, but nevertheless effectively transfers the thermal detail of the rowers in the center of the image.

The false color SE fusion results in (f) of each figure transfer virtually all RGB details while keeping the false color intact. The details are more natural and subtle than the FLIR MSX fusion results of (e), which appear to use direct edge transfer and possible edge sharpening, in comparison with the milder lookup-table-based 


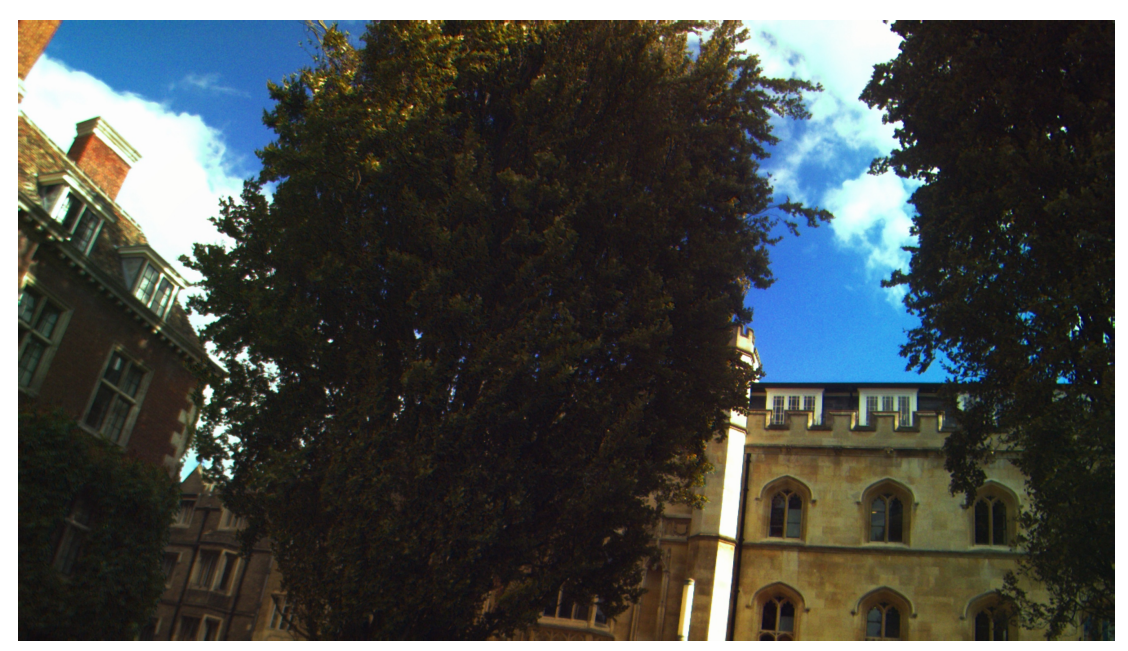

(a) RGB

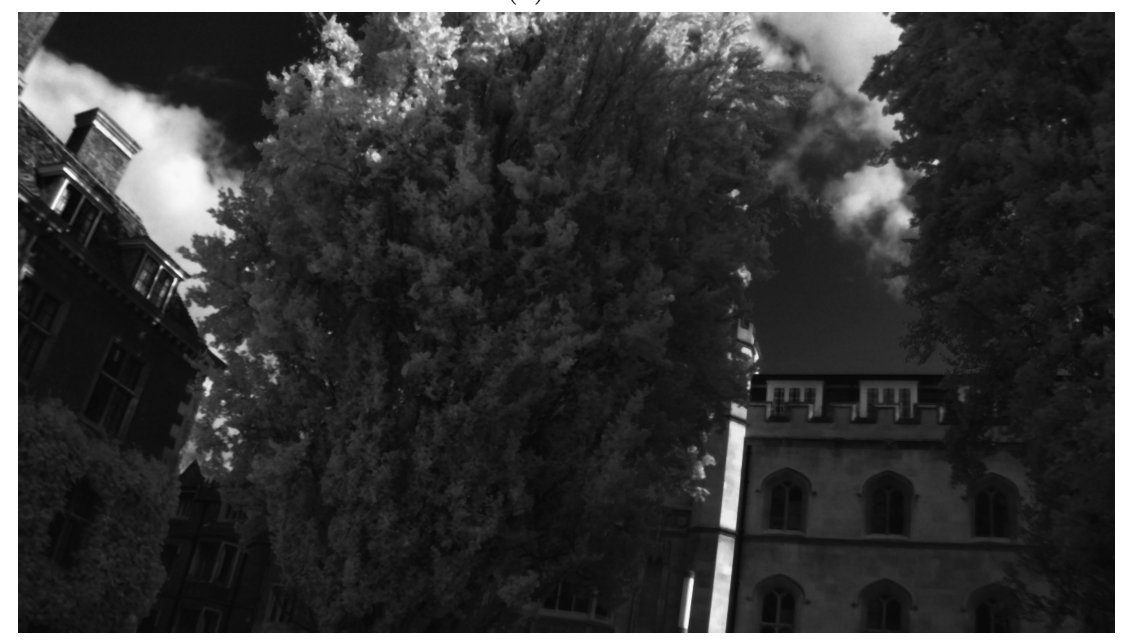

(b) NIR

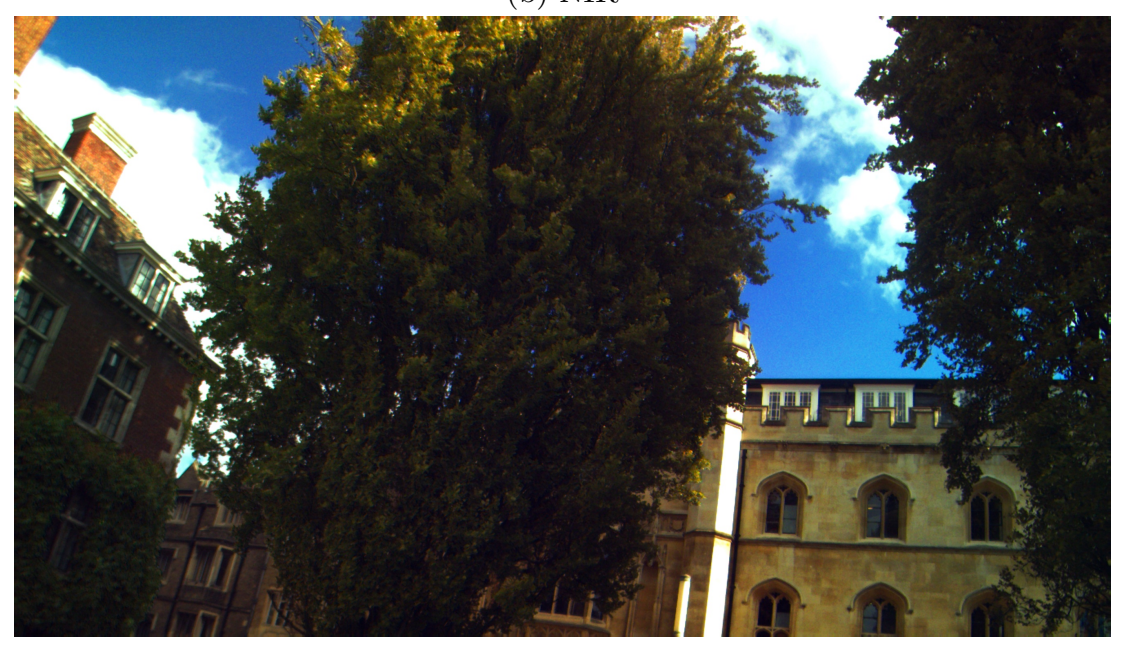

(c) $\mathrm{SE}$

Figure 3: Image fusion using an RGB-IR Bayer pattern: Cambridge street scene 1 


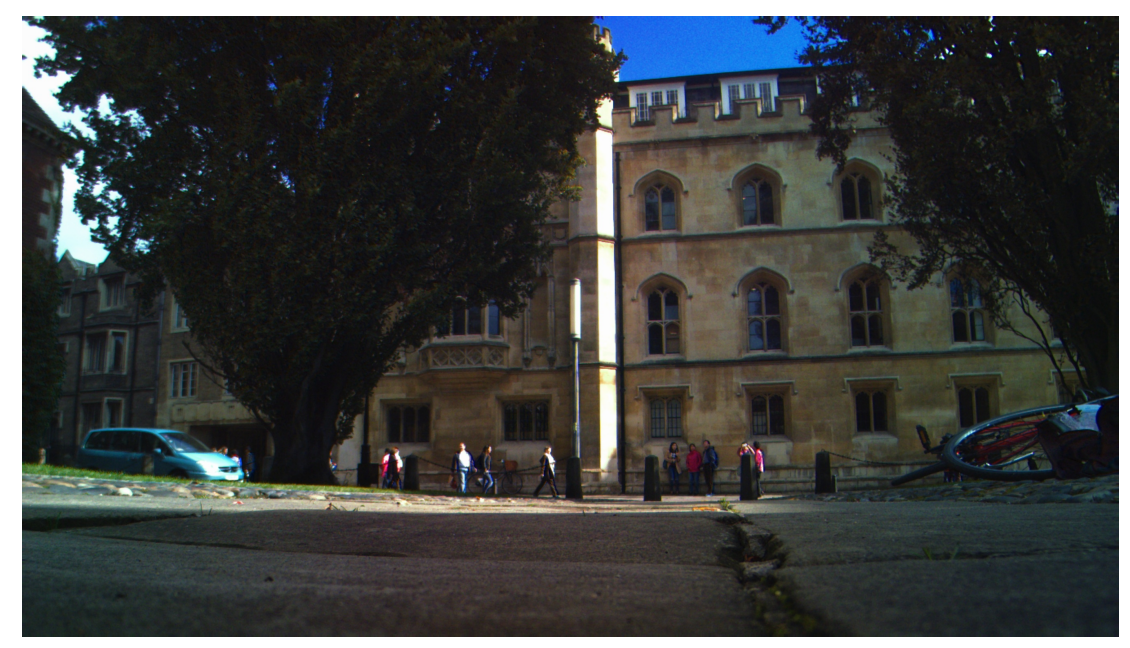

(a) RGB

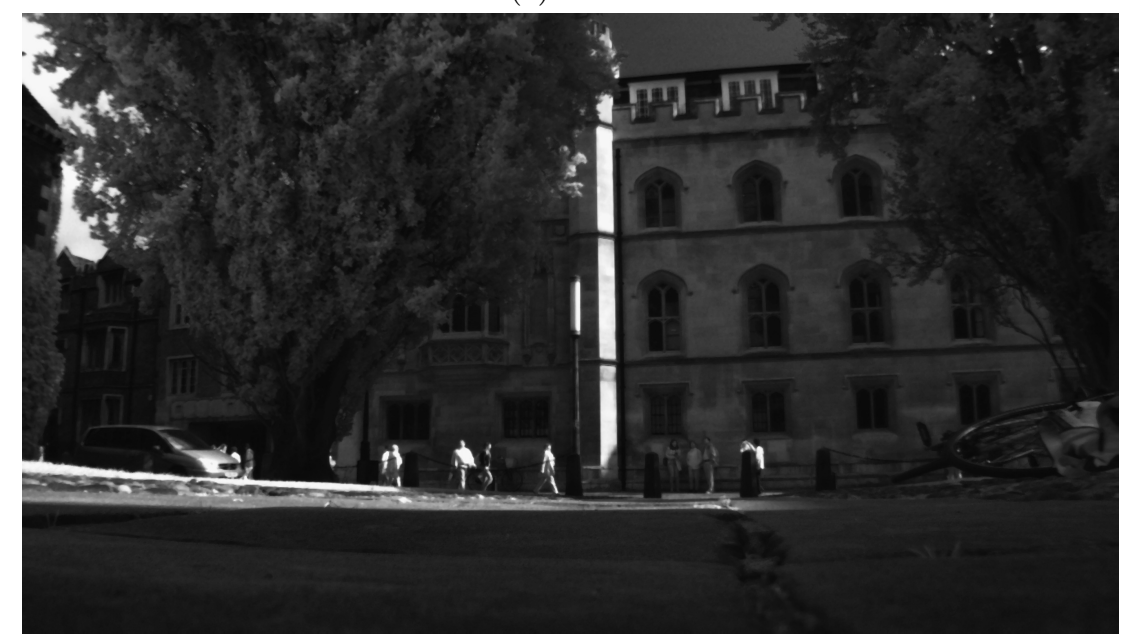

(b) NIR

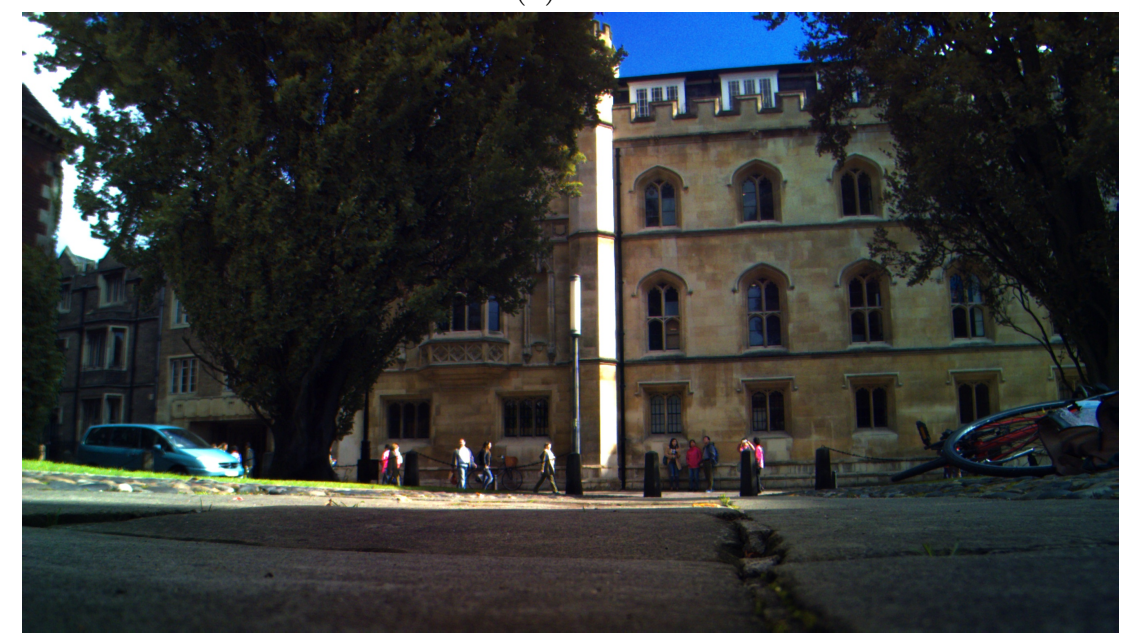

(c) SE

Figure 4: Image fusion using an RGB-IR Bayer pattern: Cambridge street scene 2 


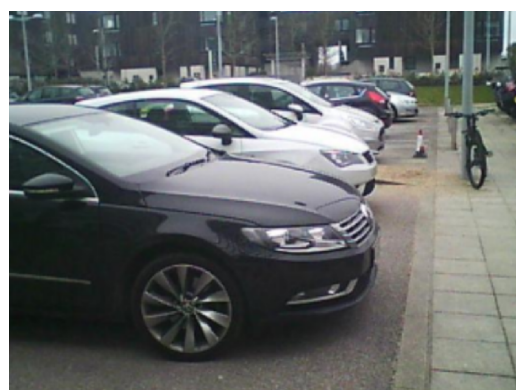

(a) RGB

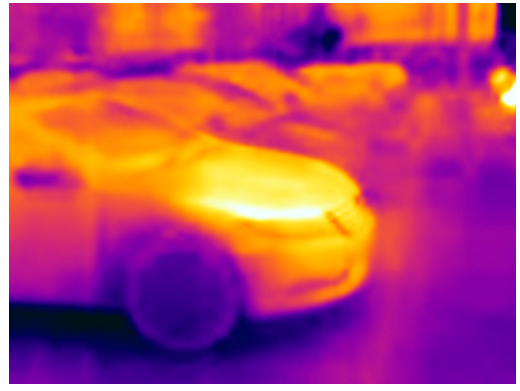

(d) Thermal (false color)

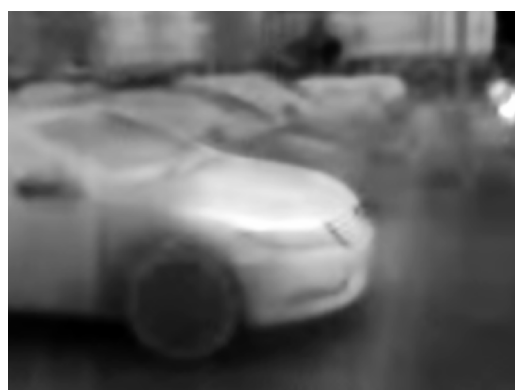

(b) Thermal

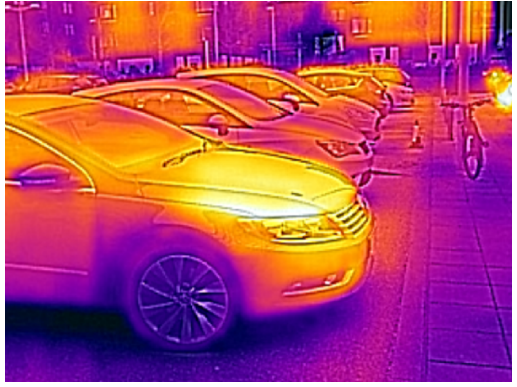

(e) FLIR MSX fusion

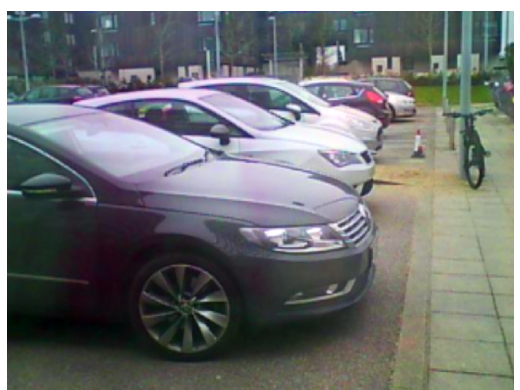

(c) SE

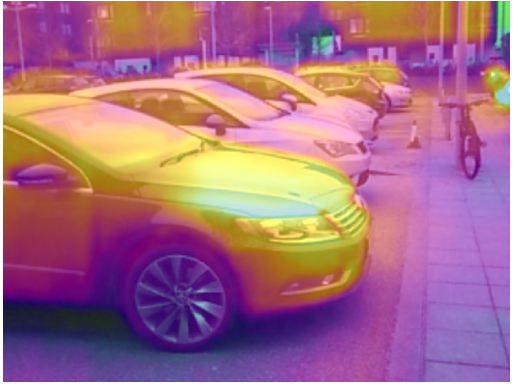

(f) SE (false color)

Figure 5: RGB-thermal image fusion using the FLIR ONE: scene 1 - cars

gradient reintegration used in the SE fusion method. Each of the two methods has their merits, and a judgment of the preferred method would have to be made depending on the specific application.

The RGB-thermal fusion shown in (c) of these figures could be integrated into a security camera for a surveillance application. A single fused image could simultaneously give a human observer both visible and thermal details, possibly requiring less attention and leading to faster object or person detection. The false color fusion shown in (f) of these figures may be a possible alternative to the current FLIR MSX fusion method used in the FLIR ONE.

\section{FUTURE WORK}

We are currently developing a real-time implementation of Spectral Edge image fusion, simultaneously capturing visible and near-infrared images and fusing them. It is already approaching real-time frame rates at $720 \mathrm{p}$ resolution. The Spectral Edge image fusion method has potential to be applied to a wide variety of commercial and scientific applications, both for single images and video.

In this paper we have used the Spectral Edge image fusion method proposed by Connah et al. ${ }^{10}$ and its iterative extension. ${ }^{11}$ We are also developing applications of the POP image fusion method, ${ }^{20}$ a new derivative based method which has the potential to increase the detail of output images due to its local processing, but does not have the color component of the Spectral Edge method. This method or others should enable even more NIR details to be transferred into the output image, while maintaining image quality.

\section{CONCLUSION}

We have demonstrated two new applications of the Spectral Edge image fusion method, which is a derivative based image fusion method integrating color information, which produces natural and detailed output images.

We have used an RGB-IR sensor to simultaneously capture visible spectrum and near-infrared images, using our own custom-designed image pipeline, before fusing the two images using Spectral Edge image fusion. This process is a form of photographic enhancement using near-infrared image information. 


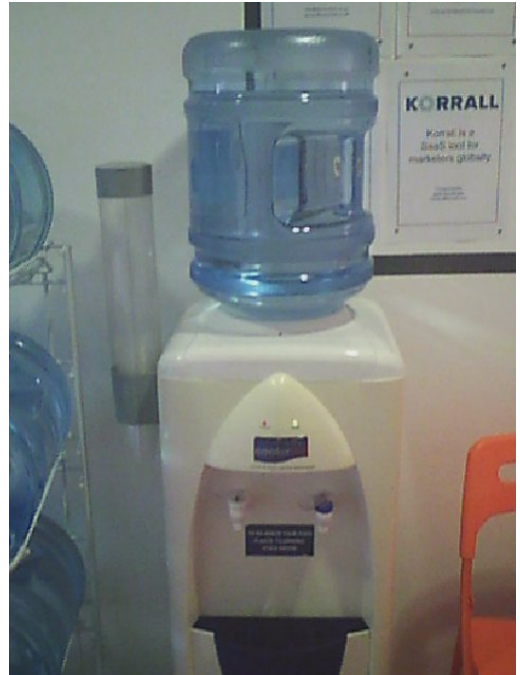

(a) RGB

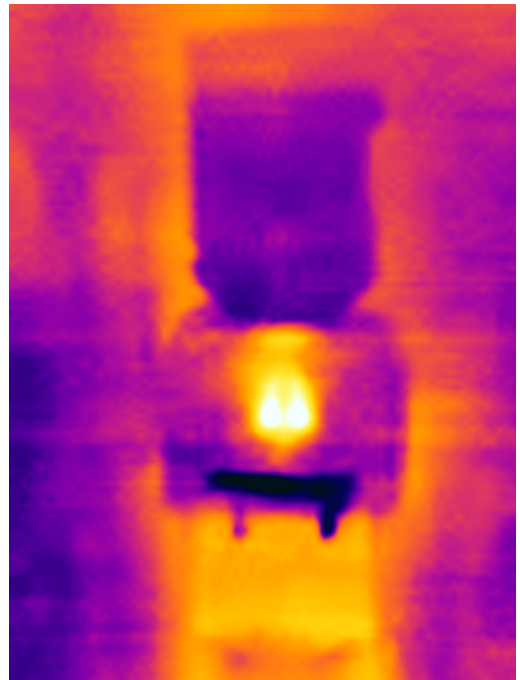

(d) Thermal (false color)

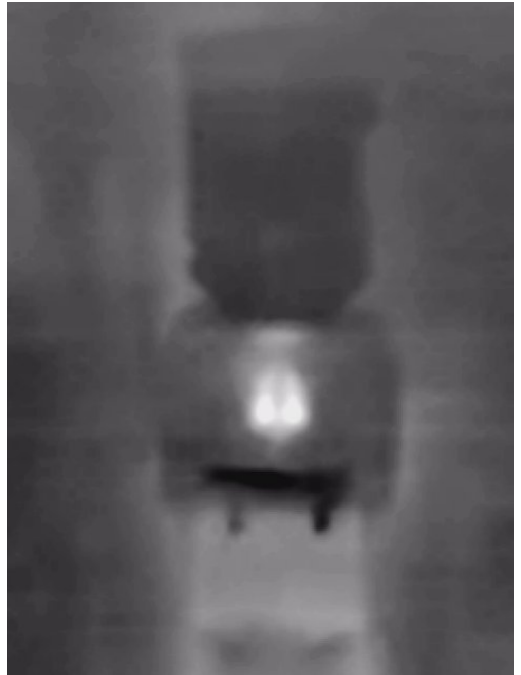

(b) Thermal

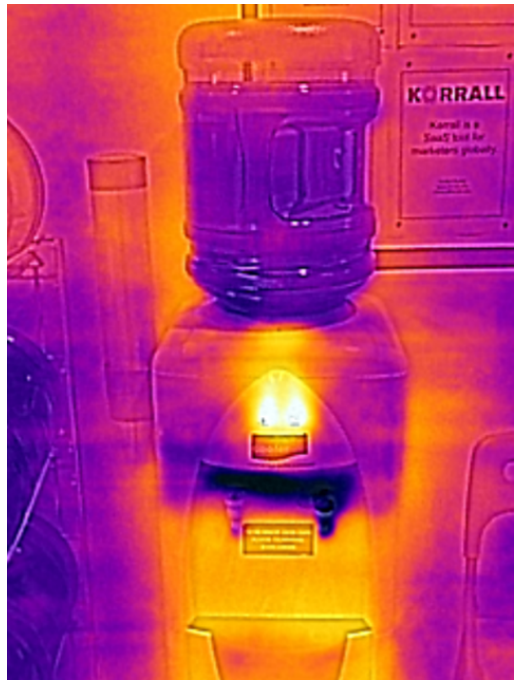

(e) FLIR MSX fusion

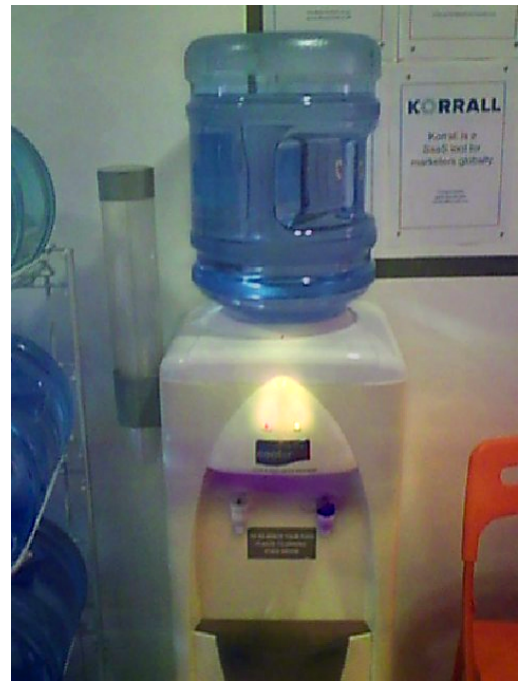

(c) SE

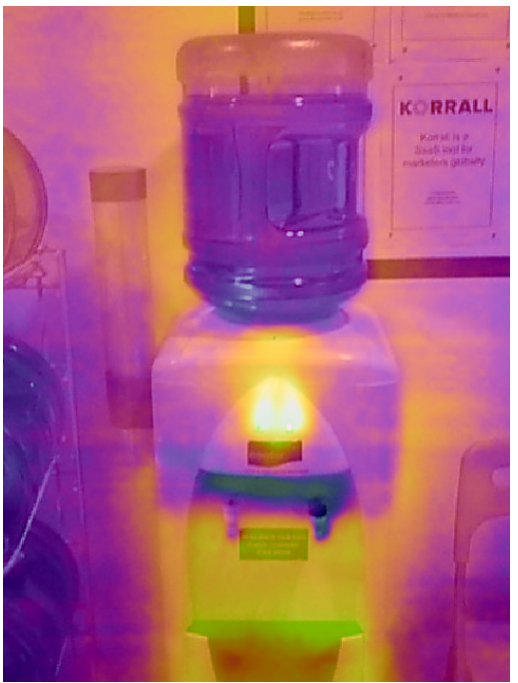

(f) SE (false color)

Figure 6: RGB-thermal image fusion using the FLIR ONE: scene 2 - water cooler 


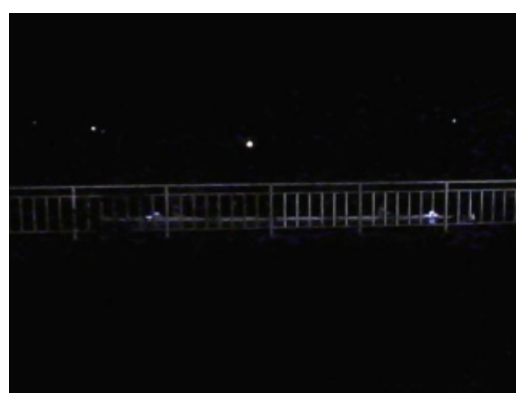

(a) RGB

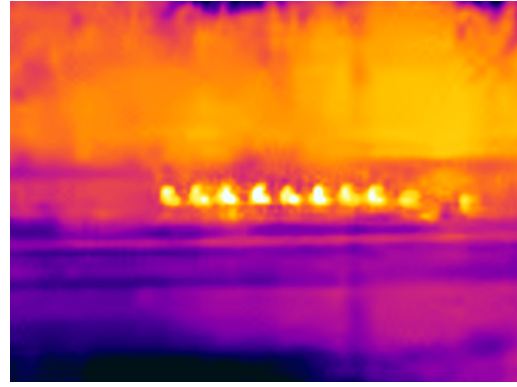

(d) Thermal (false color)

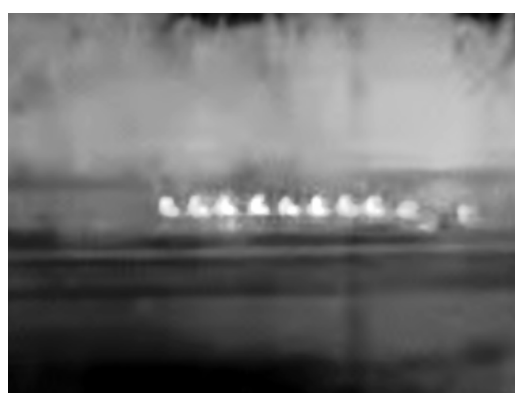

(b) Thermal

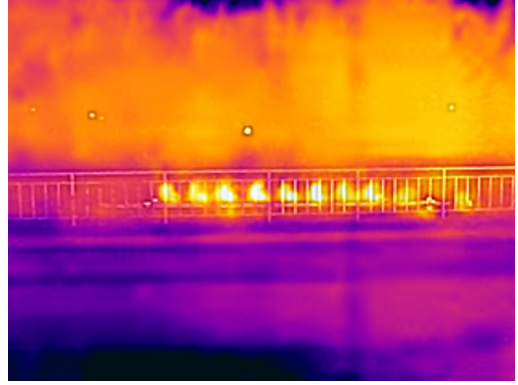

(e) FLIR MSX fusion

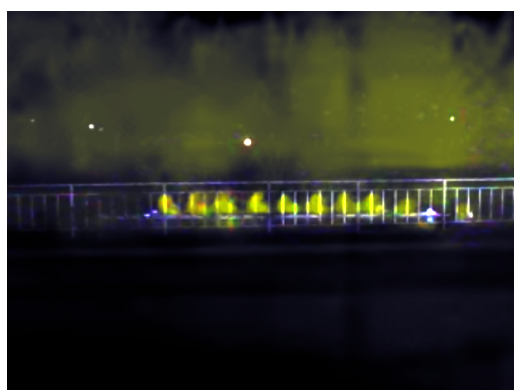

(c) SE

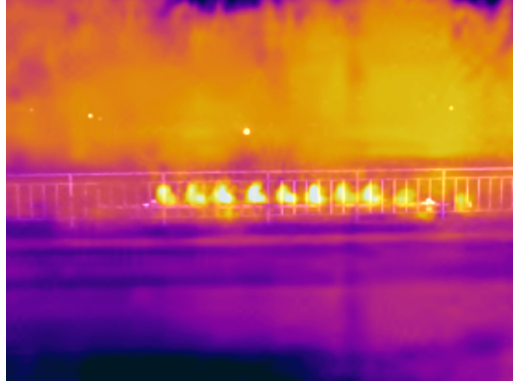

(f) SE (false color)

Figure 7: RGB-thermal image fusion using the FLIR ONE: scene 3 - rowers at night

Using the FLIR ONE smartphone-based thermal camera, we have captured visible spectrum and thermal images, and fused them using Spectral Edge image fusion. This creates an output image with both visible details and color, as well as extra details transferred from the thermal image, such as objects and people. We have also shown a false color fusion more similar to the FLIR MSX fusion technology, but which has a more subtle and natural effect.

The Spectral Edge image fusion method is a powerful technique, with many potential applications, including photographic enhancement, surveillance and remote sensing.

\section{ACKNOWLEDGMENTS}

Many thanks to Omnivision Technologies Inc., for allowing us the use of their sensor as well as lots of support.

\section{REFERENCES}

[1] Nencini, F., Garzelli, A., Baronti, S., and Alparone, L., "Remote sensing image fusion using the curvelet transform," Information Fusion 8(2), 143-156 (2007).

[2] Wang, Z. and Ma, Y., "Medical image fusion using m-pcnn," Information Fusion 9(2), 176-185 (2008).

[3] Li, S. and Yang, B., "Multifocus image fusion using region segmentation and spatial frequency," Image and Vision Computing 26(7), 971-979 (2008).

[4] Hayes, A. E., Finlayson, G. D., and Montagna, R., "RGB-NIR color image fusion: metric and psychophysical experiments," ISET T/SPIE Electronic Imaging, 93960U-93960U-9 (2015).

[5] Connah, D., Finlayson, G. D., and Bloj, M., "Seeing beyond luminance: A psychophysical comparison of techniques for converting colour images to greyscale," Color and Imaging Conference 2007(1), 336-341 (2007).

[6] Pajares, G. and De La Cruz, J. M., "A wavelet-based image fusion tutorial," Pattern Recognition 37(9), 1855-1872 (2004).

[7] Toet, A., "Image fusion by a ratio of low-pass pyramid," Pattern Recognition Letters 9(4), 245-253 (1989).

[8] Li, S., Kwok, J. T., and Wang, Y., "Multifocus image fusion using artificial neural networks," Pattern Recognition Letters 23(8), 985-997 (2002). 
[9] Socolinsky, D. A. and Wolff, L. B., "Multispectral image visualization through first-order fusion," Image Processing, IEEE Transactions on 11(8), 923-931 (2002).

[10] Connah, D., Drew, M. S., and Finlayson, G. D., "Spectral Edge image fusion: Theory and applications," Computer Vision, European Conference on , 65-80 (2014).

[11] Finlayson, G. D. and Hayes, A. E., "Iterative Spectral Edge image fusion," Color and Imaging Conference 2015(1), 41-45 (2015).

[12] Di Zenzo, S., "A note on the gradient of a multi-image," Computer vision, Graphics, and Image Processing 33(1), 116-125 (1986).

[13] Finlayson, G. D., Connah, D., and Drew, M. S., "Lookup-table-based gradient field reconstruction," Image Processing, IEEE Transactions on 20(10), 2827-2836 (2011).

[14] "Omnivision OV4682 RGB-IR sensor." http://www.ovt.com/products/sensor.php?id=145. Accessed: 2016-03-08.

[15] Brown, M. and Susstrunk, S., "Multi-spectral sift for scene category recognition," Computer Vision and Pattern Recognition, IEEE Conference on , 177-184 (2011).

[16] Lin, C., "Pixel grouping." https://sites.google.com/site/chklin/demosaic. Accessed: 2016-03-08.

[17] Finlayson, G. D. and Trezzi, E., "Shades of gray and colour constancy," Color and Imaging Conference 2004(1), 37-41 (2004).

[18] "FLIR ONE." http://www.flir.co.uk/flirone/. Accessed: 2016-03-08.

[19] Strandemar, K., "Infrared resolution and contrast enhancement with fusion," Patent 9,171,361 (2015).

[20] Finlayson, G. D. and Hayes, A. E., "Pop image fusion - derivative domain image fusion without reintegration," The IEEE International Conference on Computer Vision (ICCV) , 334-342 (December 2015). 